> A la complexité déjà existante des mécanismes immunitaires impliqués dans l'infection par le parasite Plasmodium s'ajoute un mécanisme décrit récemment par notre équipe et qui rapporte l'implication de l'histamine, une amine vasoactive, dans la pathogenèse du paludisme. Cette étude repose sur deux approches complémentaires : une approche génétique qui consiste à utiliser des souris déficientes en récepteurs histaminiques (HR1, HR2, HR3) ou en histamine, et une approche pharmacologique consistant à utiliser des antagonistes pharmacologiques de ces récepteurs. Les résultats obtenus montrent que des processus reconnus comme étant associés à la pathogenèse du neuropaludisme chez la souris tels que la rupture de la barrière hématoencéphalique, la séquestration de globules rouges et l'adhésion de lymphocytes T à l'endothélium des capillaires cérébraux, sont associés à la production d'histamine. Ces travaux ouvrent de nouvelles perspectives pour une meilleure prise en charge thérapeutique du paludisme chez l'homme. <

\title{
Rôle de l'histamine et des récepteurs histaminiques dans la pathogenèse du paludisme
}

\author{
Walid Beghdadi, Adeline Porcherie, \\ Bradley S. Schneider, David Dubayle, \\ Roger Peronet, Michel Huerre, Takeshi Watanabe, \\ Hiroshi Ohtsu, Jacques Louis, Salah Mécheri
}

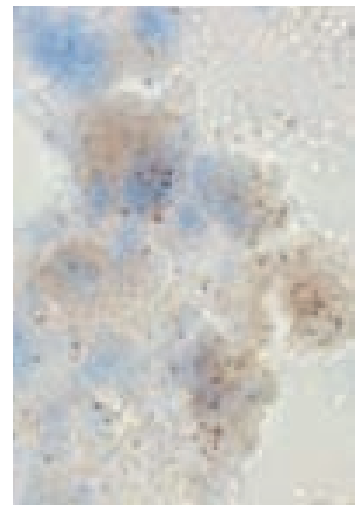

que le Royaume-Uni, l'Allemagne, I'Italie, l'Espagne, les Pays-Bas et la Suisse. Les ÉtatsUnis déclarent quant à eux environ 1500 cas annuels [3]. Cependant, le nombre total de cas de paludisme importés en France a diminué ces deux dernières années (5 267 cas estimés en 2006 contre 4404 en 2007) $[4,5]$.

Le neuropaludisme est un syndrome clinique caractérisé par des manifestations neurologiques graves induites par Plasmodium falciparum chez l'homme et qui restent à l'heure actuelle très difficiles à expliquer. II peut aussi être induit par une souche bien particulière de Plasmodium bergheï ( $P b A N K A)$ chez les rongeurs.

L'étude des mécanismes physiopathologiques étant complexe chez l'homme, les modèles expérimentaux de neuropaludisme chez la souris constituent actuellement l'un des moyens pour aborder et comprendre ces mécanismes. Les modèles in vivo permettent d'étudier les 
processus physiopathologiques, de mettre en évidence de nouveaux facteurs et de nouveaux mécanismes. Les souris des souches CBA ou C57BL/6 infectées avec $P b$ ANKA représentent les deux modèles expérimentaux murins de neuropaludisme les plus utilisés et sont considérées comme des souris susceptibles d'être infectées. En revanche, les souris BALB/c sont considérées comme des souris résistantes au développement du neuropaludisme.

Trois hypothèses ont été émises afin d'expliquer la neuropathogenèse chez l'homme: la théorie de la perméabilité fondée sur une rupture de la barrière hémato-encéphalique (BHE), la théorie mécanique basée essentiellement sur la cytoadhérence des globules rouges infectés à l'endothélium vasculaire, et enfin la théorie immunologique qui implique les réponses immunitaires antipalustres dans la neuropathogenèse [6]. Une façon d'aborder la complexité des processus physiopathologiques du neuropaludisme est de proposer un mécanisme unifiant ces hypothèses et qui repose sur l'idée qu'une réponse inflammatoire d'un type particulier serait responsable de la pathogenèse de cette maladie. L'hypothèse que nous proposons réside dans le fait qu'une réponse de type allergique, à l'issue de laquelle des médiateurs inflammatoires comme l'histamine peuvent être générés, fait suite à l'activation immunologique (Ig£-dépendante) ou non (Ig£-indépendante) des mastocytes et des basophiles par des constituants parasitaires. Les activités biologiques de l'histamine en font un candidat de choix étant donné ses propriétés hémodynamiques, pro-inflammatoires et immunorégulatrices.

\section{Propriétés biologiques de l'histamine et de ses récepteurs}

L'histamine est un médiateur chimique stocké dans les granules intracytoplasmiques des mastocytes et des basophiles puis libéré en réponse à différents stimulus et particulièrement lors de réactions d'hypersensibilité de type immédiat dépendantes de l'Igع. Cette molécule est synthétisée par décarboxylation de l'histidine sous l'action de l'histidine décarboxylase (HDC). L'histamine exerce ses effets via l'activation de quatre récepteurs histaminiques $\mathrm{H} 1, \mathrm{H} 2, \mathrm{H} 3$ et $\mathrm{H} 4$ qui sont exprimés à la surface de différentes cellules, telles que les cellules musculaires lisses, les monocytes, les cellules immunocompétentes ou inflammatoires, les cellules endothéliales ou épithéliales et les fibres nerveuses. Selon le type de récepteur histaminique prédominant et le système expérimental étudié, l'histamine peut avoir des effets pro- ou anti-inflammatoires.

L'activation du récepteur $\mathrm{Hl}$ engendre la plupart des effets proinflammatoires de l'histamine mais aussi la polarisation des divers aspects de la réponse immune spécifique en modulant la balance Thl/ Th2 [7]. L'augmentation de l'expression de la P-sélectine (CD62P), qui favorise l'adhésion initiale des monocytes à l'endothélium vasculaire suite à un traitement avec l'histamine, représente un mécanisme important dans la migration des monocytes vers les barrières endothéliales [8]. L'utilisation d'agonistes ou d'antagonistes des récepteurs $\mathrm{Hl}$ et $\mathrm{H} 2$ contribue de manière importante aux modifications des mécanismes de défense contre les agents pathogènes. La stimulation des cellules endothéliales vasculaires via les récepteurs $\mathrm{Hl}$ conduit à l'augmentation de la perméabilité vasculaire (en particulier dans les veinules postcapillaires) [9] et à la libération de NO (nitric oxide) [10]. Par ailleurs, le traitement avec un antagoniste du récepteur $\mathrm{H} 2$ semble inhiber l'expression de certaines molécules d'adhésion telles que ICAM-1 (intercellular adhesion molecule) et la sélectine $P$, et diminuer l'adhésion des polynucléaires neutrophiles [11] suggérant un rôle pro-inflammatoire de l'histamine via le récepteur $\mathrm{H} 2$.

Les effets immunosuppresseurs et antiinflammatoires de l'histamine, tels que l'inhibition du chimiotactisme des polynucléaires neutrophiles [12], la sécrétion d'IL(interleukine)-12 par les monocytes [13], ou encore l'induction de la production d'IL-10 [13], sont pour la plupart le résultat de la stimulation des récepteurs $\mathrm{H} 2$. Le récepteur $\mathrm{H3}$ a été identifié en 1983 [14], à la fois dans le système nerveux central et périphérique, comme un récepteur présynaptique qui inhibe la synthèse et la libération d'histamine ainsi que d'autres neurotransmetteurs comme la dopamine, la sérotonine ou l'acétylcholine [14]. Il est exprimé dans les fibres nerveuses et sa stimulation par l'histamine diminue la libération d'histamine mais aussi d'autres neuromédiateurs [15]. L'activation du récepteur $H 4$, exprimé par les cellules mononucléées et les lymphocytes $T$ CD4 $4^{+}$et $C D 8^{+}[16]$, inhibe la production de la chimiokine CCL2 engendrant ainsi une réduction du recrutement des monocytes [17].

L'histamine fait l'objet de recherches intenses et son étude est capitale pour mieux comprendre et traiter l'allergie. Par ailleurs, certains travaux s'intéressent à d'autres fonctions. Il est bien connu que cette molécule est impliquée dans des maladies gastriques (I'ulcère gastro-duodénal par exemple) et qu'elle sert de neuromédiateur dans le cerveau. Cette dernière propriété a un intérêt particulier dans les travaux sur le neuropaludisme.

\section{Relations entre histamine et infection par Plasmodium}

L'augmentation des niveaux d'histamine dans le plasma et dans les tissus, provenant de basophiles et mastocytes, a été associée à la sévérité de la maladie au cours des infections à Plasmodium falciparum chez l'homme et dans plusieurs modèles d'infections par Plasmodium chez les animaux $[18,19]$. En outre, des concentrations plasmatiques élevées d'anticorps Ig $\operatorname{la}$, qui se lient aux basophiles et aux mastocytes et qui peuvent déclencher la libération d'histamine, ont été associées à la gravité de l'infection par Plasmodium falciparum [20]. Outre la libération d'histamine déclenchée par les Igદ, le HRF (histamine-releasing factor), un peptide décrit chez l'homme et la souris, provoque la libération d'histamine, d'IL-4, et d'IL-13 par les basophiles [21]. Ce peptide appartient à une famille de protéines appelées protéines de tumeurs contrôlées au niveau traductionnel (TCTP). Récemment, un 
TCTP présentant une forte homologie avec le HRF humain a été identifié chez Plasmodium falciparum [22]. Le TCTP de Plasmodium falciparum a pu être mis en évidence dans le plasma de patients infectés et son activité histaminolibératrice sur les basophiles humains a pu être démontrée [23], suggérant que le TCTP de Plasmodium falciparum peut affecter la réponse immunitaire de l'hôte en influant sur les réponses in vivo par sa capacité à libérer l'histamine [23]. Il convient de noter que depuis le début de l'infection par Plasmodium (piqûre infectante de moustique) et tout au long de l'infection (parasitémie), les vaisseaux sanguins et les cellules immunitaires sont constamment stimulés par l'histamine. L'interférence avec les effets biologiques de l'histamine pourrait représenter une nouvelle stratégie visant à manipuler la réponse immunitaire contre le parasite Plasmodium.

\section{Rôle de la signalisation dépendante de l'histamine dans la pathogenèse du paludisme}

L'effet délétère de l'histamine produite au cours de l'infection par Plasmodium a été bien étudié dans un modèle d'infection par le parasite $P$ bergheï NK65 ( $P b$ NK65), souche létale n'entraînant pas de symptômes de neuropaludisme. Le rôle plus spécifique de l'histamine dans la neuropathogenèse a été élucidé par l'examen des mécanismes physiopathologiques impliqués dans l'infection en utilisant la souche létale $P b$ ANKA responsable du neuropaludisme dans des modèles de souris sauvages ou déficientes en histamine.

\section{Rôle de l'histamine et des récepteurs histaminiques dans l'infection par Pb NK65}

Ces études ont été abordées par une approche pharmacologique qui consiste à utiliser des antagonistes des

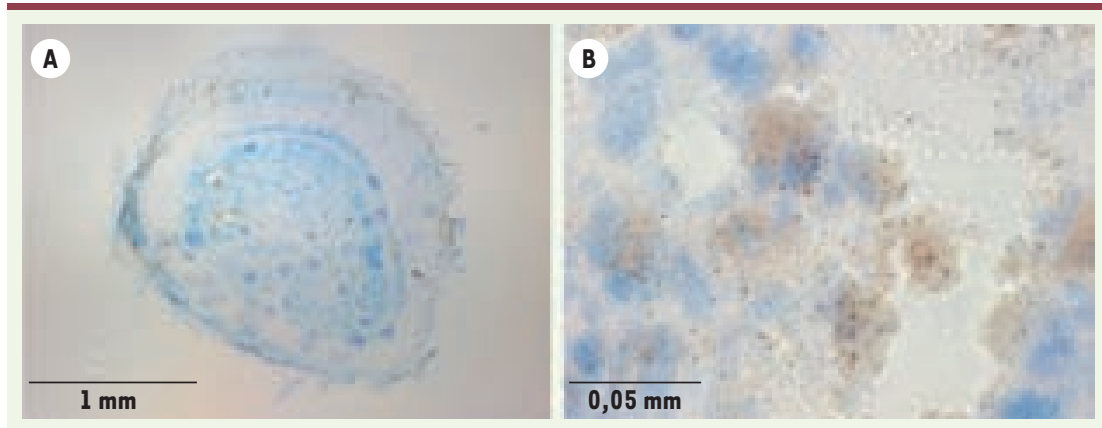

Figure 1. Mise en évidence d'agrégats de globules rouges dans le cerveau de souris C57BL/6 6 jours après infection avec Plasmodium bergheï ANKA. A. Dépôts de globules rouges infectés $(x 40)$. B. Les mêmes érythrocytes infectés observés à un plus fort grossissement (x600) où on peut distinguer des dépôts d'hémozoïne. Dans les mêmes expériences, les souris HDC-KO déficientes en histamine ne montrent aucun dépôt d'agrégats. récepteurs histaminiques, complétée par une approche génétique utilisant des souris dont les gènes codant pour les récepteurs $\mathrm{Hl}\left(H I R^{-/-}\right)$ et les récepteurs $\mathrm{H} 2\left(\mathrm{H}_{2} \mathrm{R}^{-/-}\right)$ont été invalidés. Afin de déterminer le rôle direct de l'histamine dans la pathogenèse du paludisme, des souris déficientes en histamine par délétion du gène codant pour l'histidine décarboxylase (HDC) ont été également examinées.

Le traitement avec des antagonistes des récepteurs histaminiques $\mathrm{Hl}$ et $\mathrm{H} 2$ de souris infectées par la souche $\mathrm{Pb}$ NK65 assure une survie prolongée de ces souris contrairement aux souris sauvages non traitées. Les parasitémies des souris infectées traitées ou non par les antagonistes des récepteurs histaminiques ne sont pas différentes de manière significative [24].

Afin de confirmer l'implication des récepteurs $\mathrm{Hl}$ et $\mathrm{H} 2$ dans la pathogenèse du paludisme, des souris déficientes en récepteurs $\mathrm{Hl}$ ou $\mathrm{H} 2$ de l'histamine ont été infectées puis leur survie a été comparée à celle des souris sauvages C57BL/6. Alors qu'aucune différence significative n'a été observée entre les différents groupes de souris en ce qui concerne la parasitémie, le suivi de la mortalité des souris révèle une résistance accrue des souris déficientes pour les récepteurs $\mathrm{Hl}$ et $\mathrm{H} 2$ (délai de mortalité retardé de 3 jours en moyenne) contrairement aux souris sauvages $\mathrm{C} 57 \mathrm{BL} / 6$. Lorsque des souris déficientes en histamine ( $\left.H D C^{-/-}\right)$ont été infectées dans les mêmes conditions, celles-ci se sont avérées beaucoup plus résistantes que les souris sauvages et les souris individuellement déficientes en récepteur $\mathrm{Hl}$ ou $\mathrm{H} 2$ [24].

\section{Rôle de l'histamine dans la pathogenèse du neuropaludisme}

L'implication directe de l'histamine dans la neuropathogenèse au cours de l'infection a été démontrée par la survie des souris $H_{D C} C^{-/}$et l'absence de signes cliniques de neuropaludisme après une infection par la souche $\mathrm{Pb}$ ANKA. Les souris meurent néanmoins très tardivement d'hyperparasitémie (30-35 jours après infection au lieu de 8 à 10 jours). Plusieurs théories ont été proposées pour expliquer la survenue du neuropaludisme chez l'homme: la théorie mécanique, la théorie immunologique et enfin la théorie de la perméabilité. Cette dernière est fondée sur la perméabilité de la barrière hématoencéphalique (BHE) qui constitue l'un des mécanismes hémodynamiques liés à l'histamine [25] et associés au développement du neuropaludisme chez l'homme [26]. La résistance des souris $H D C^{-/-}$vis-à-vis du parasite $P b$ ANKA est clairement associée à la préservation de la BHE contrairement aux souris C57BL/6 qui présentent une rupture de la $B H \varepsilon$ associée à une séquestration de globules rouges infectés dans le cerveau de celles-ci [24] (Figure 1). Une des caractéristiques du neuropaludisme chez la souris est la séquestration des lymphocytes $\mathrm{T} \mathrm{CD}^{+}$et $\mathrm{CD} 8^{+}$ dans les microvaisseaux sanguins du cerveau [27, 28]. Cela a été confirmé par nos 
résultats qui montrent une augmentation significative des lymphocytes $T$ $\mathrm{CD}^{+}$et $\mathrm{CD} 8^{+}$dans le cerveau des souris $\mathrm{C} 57 \mathrm{BL} / 6$ infectées par $\mathrm{Pb}$ ANKA. Une telle séquestration n'a pas été observée dans le cerveau des souris $H D C^{-/-}$ infectées [24]. Ces résultats suggèrent que l'histamine produite au cours de l'infection par $\mathrm{Pb} A N K A$ induit une réponse inflammatoire engendrant la séquestration des lymphocytes $T$ dans les capillaires sanguins du cerveau, ce qui favorise le développement du neuropaludisme. Un schéma illustrant notre vision du phénomène est représenté sur la Figure 2. Dans ce contexte, il a été rapporté que l'histamine est essentielle au recrutement des lymphocytes $\mathrm{T} \mathrm{CD}^{+}$et $\mathrm{CD} 8^{+}$spécifiques de l'antigène [29]. De nombreuses études ont démontré le rôle essentiel de la molécule d'adhésion ICAM-1 dans la séquestration des globules rouges infectés, et par conséquent dans le développement de la pathogenèse du paludisme, notamment par l'utilisation de souris déficientes en ICAM-1, qui sont résistantes au neuropaludisme $[30,33]$. Nos résultats confortent l'implication de l'expression d'ICAM-1 dans la pathogenèse du neuropaludisme. La faible expression d'ICAM-1 à la surface des cellules endothéliales vasculaires du cerveau de souris $H D C^{-1-}$ peut expliquer l'absence de séquestration des lymphocytes $T$ dans le cerveau de ces souris. II est intéressant de noter une réduction de la synthèse d'ICAM-1 chez les souris $H D C^{-1-}$ qui présentent un profil de résistance similaire à celui des souris $I C A M-1^{-/-}$, suggérant ainsi un mécanisme de contrôle de l'histamine sur l'expression d'ICAM-1. En effet, il a été rapporté que l'histamine stimule l'expression d'ICAM-1 et VCAM-1 (vascular cell adhesion molecule) par les cellules endothéliales [31] et que la cétirizine, un antagoniste du récepteur $\mathrm{Hl}$, a été identifiée comme une molécule possédant des propriétés anti-inflammatoires par sa capacité d'inhibition de recrutement et d'activation de leucocytes et par la réduction de l'expression d'ICAM-1 sur les kératinocytes [32].

\section{Conclusion}

En résumé, les résultats présentés dans cette revue sont en accord avec des données publiées précédemment

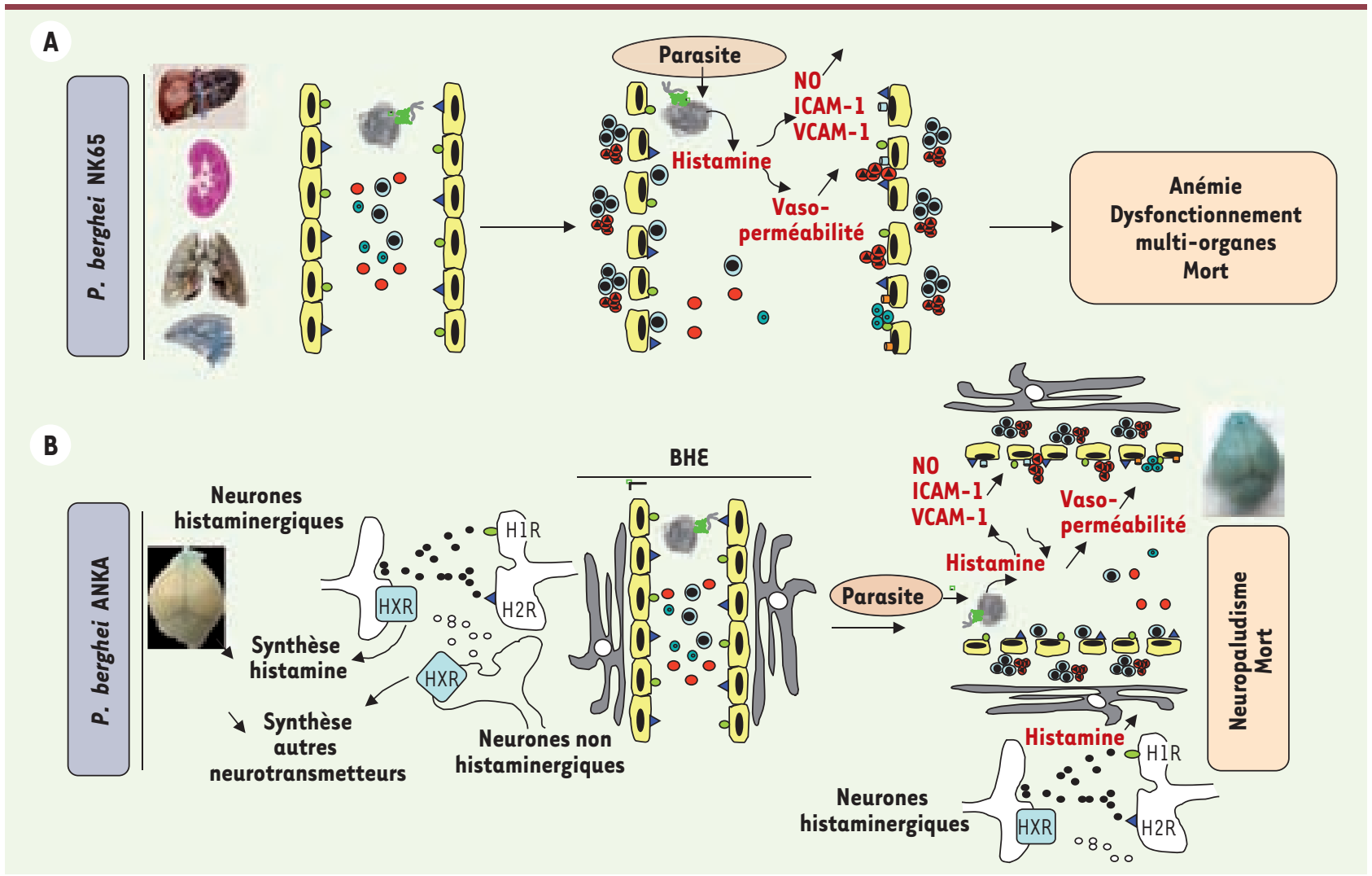

Figure 2. Représentation schématique du rôle de l'histamine. L'histamine est impliquée dans l'augmentation de la perméabilité capillaire (A) et la rupture de la barrière hémato-encéphalique (BHE) (extravasation du bleu Evans) (B) à la suite de l'infection par Plasmodium bergheï souche NK65 (souche létale mais sans signes neurologiques) ( $A$ ) ou par Pb ANKA (souche létale génératrice de neuropaludisme) (B). Dans les deux cas, l'histamine, libérée par des mécanismes qui restent à identifier, est à l'origine de l'augmentation de la synthèse de NO, d'une surexpression de molécules d'adhésion ICAM-1 et VCAM- 1 ainsi qu'une augmentation de la perméabilité capillaire. Il en découle une séquestration au niveau des endothéliums capillaires périphériques ( $A$ ) ou centraux (B) de globules rouges infectés ainsi que de lymphocytes T CD4 et CD8. Au niveau central, l'origine de l'histamine peut être périphérique ou centrale. Dans ce dernier cas, la synthèse et la libération de l'histamine, qui est également un neurotransmetteur, sont sous le contrôle de facteurs de nature encore indéfinie (HXR). 
démontrant l'association entre les niveaux d'histamine produite au cours de l'infection et la sévérité du paludisme chez l'homme et la souris. Ces résultats sont également en accord avec le concept selon lequel des mécanismes pathogéniques du paludisme grave serait lié à un défaut de contrôle d'une réponse inflammatoire excessive. Cela ouvre des perspectives nouvelles en termes d'approches thérapeutiques puisque, aussi bien dans les modèles murins de neuropaludisme que dans des essais cliniques chez l'homme, l'utilisation d'antihistaminiques $\mathrm{Hl}$ semble montrer une certaine efficacité. Les mécanismes mettant en jeu l'histamine révèlent également le rôle possible de la réponse allergique dans la pathogenèse du paludisme. De ce point de vue, la caractérisation des mécanismes de la réponse inflammatoire allergique au cours de l'infection palustre est une voie d'investigation prometteuse dans le futur. $\diamond$

\section{SUMMARY}

Role of histamine and histamine receptors in the pathogenesis of Malaria

A hallmark of the host response to Plasmodium parasite is an inflammatory reaction characterized by elevated histaminemia levels. Since histamine, which acts through four different receptors and which synthesis is under the control of the histidine decarboxylase (HDC), is endowed with pro-inflammatory and immunosuppressive activities, we hypothesized that this vaso-active amine may participe to malaria pathogenesis. Combining genetic and pharmacologic approaches by using $\mathrm{HIR}^{-/-}$, $\mathrm{H}_{2} \mathrm{R}^{-/-}, \mathrm{H}_{3} \mathrm{R}^{-/-}, \mathrm{HDC}^{-/-}$mice and $\mathrm{H} 1 \mathrm{R}, \mathrm{H} 2 \mathrm{R}-$, and H3Rantagonists, respectively, we found that cerebral malaria-associated pathogenetic processes such as blood brain barrier disruption, and Tymphocyte sequestration to cerebral vascular endothelium in mice were associated with histamine production. The identification of this novel inflammatory pathway and its implication in Plasmodium infection may lead to novel strategies to manipulate the anti-Plasmodium immune response and may provide new therapeutic tools to alleviate malaria disease. $\diamond$

\section{RÉFÉRENCES}

1. Hay SI, Guerra CA, Tatem AJ, et al. The global distribution and population at risk of malaria : past, present, and future. Lancet Infect Dis 2004 ; $4: 327-36$.

2. Wongsrichanalai C, Pickard AL, Wernsdorfer WH, Meshnick SR. Epidemiology of drug-resistant malaria. Lancet Infect Dis 2002 ; 2: 209-18.

3. Legros F, Amaud A, દl Mimouni B, Danis M, et CNREPIA. Paludisme d'importation en France métropolitaine: données épidémiologiques 2001-2004. Bull Epidemiol Hebd 2006; 32.

4. Rapports d'activités 2006 du Centre national de référence sur le paludisme. www.imea.fr/imea-recherche/imea-rba-chimiosenspalu.php
5. Rapports d'activités 2007 du Centre national de référence sur le paludisme. www.imea.fr/ imea-recherche/imea-rba-chimiosenspalu.php

6. Van der Heyde HC, Nolan J, Combes V, et al. A unified hypothesis for the genesis of cerebral malaria : sequestration, inflammation and hemostasis leading to microcirculatory dysfunction. Trends Parasitol $2006 ; 22: 503-8$.

7. Simons FE. Advances in Hl-antihistamines. N Engl J Med 2004 ; 351 : 2203-17.

8. Da Costa Martins P, Garcia-Vallejo JJ, van Thienen JV, et al. P-selectin glycoprotein ligand-1 is expressed on endothelial cells and mediates monocyte adhesion to activated endothelium. Arterioscler Thromb Vasc Biol 2007 ; 27 : 1023-9.

9. Svensjo $\varepsilon$, Grega GJ. Evidence for endothelial cell-mediated regulation of macromolecular permeability by postcapillary venules. Fed Proc $1986 ; 45: 89-95$.

10. Van De Voorde J, Leusen I. Role of the endothelium in the vasodilator response of rat thoracic aorta to histamine. Eur J Pharmacol $1993 ; 87: 113-20$.

11. Takeuchi $\mathrm{Y}$, Okayama N, Imaeda K, et al. Effects of histamine 2 receptor antagonists on endothelial-neutrophil adhesion and surface expression of endothelial adhesion molecules induced by high glucose levels. J Diabetes Complications $2007 ; 21: 50-5$.

12. Bury TB, Corhay JL, Radermecker MF. Histamine-induced inhibition of neutrophil chemotaxis and T-lymphocyte proliferation in man. Allergy $1992 ; 47: 624-9$.

13. Elenkov IJ, Webster $\varepsilon$, Papanicolaou DA, et al. Histamine potently suppresses human IL-12 and stimulates IL-10 production via H2 receptors. J Immunol 1998 ; 161 : 2586-93.

14. Arrang JM, Garbarg M, Schwartz JC. Auto-inhibition of brain histamine release mediated by a novel class (H3) of histamine receptor. Nature $1983 ; 302: 832-7$.

15. Haas HL, Sergeeva OA, Selbach 0. Histamine in the nervous system. Physiol Rev 2008 ; $88: 1183-241$.

16. Zhu Y, Michalovich D, Wu H, et al. Cloning, expression, and pharmacological characterization of a novel human histamine receptor. Mol Pharmacol $2001 ; 59: 434-41$.

17. Dijkstra D, Leurs R, Chazot $P$, et al. Histamine downregulates monocyte CCL2 production through the histamine H4 receptor. J Allergy Clin Immunol 2007 ; 120 : 300-7.

18. Bhattacharya U, Roy S, Kar PK, et al. Histamine and kinin system in experimental malaria. Indian J Med Res $1988 ; 88:$ 558-63.

19. Srichaikul T, Archararit N, Siriasawakul T, Viriyapanich T. Histamine changes in Plasmodium falciparum malaria. Trans R Soc Trop Med Hyg 1976; 70 : 36-8.

20. Perlmann P, Perlmann H, દIGhazali G, Blomberg MT. Ig $\varepsilon$ and tumor necrosis factor in malaria infection. Immunol Lett $1999 ; 65: 29-33$.

21. MacDonald SM, Rafnar T, Langdon J, Lichtenstein LM. Molecular identification of an IgE-dependent histamine-releasing factor. Science $1995 ; 269: 688-90$.

22. Bhisutthibhan J, Pan XP, Hossler PA, et al. The Plasmodium falciparum translationally controlled tumor protein homolog and its reaction with the antimalarial drug artemisinin. J Biol Chem $1998 ; 273: 16192-8$.

23. MacDonald SM, Bhisutthibhan J, Shapiro TA, et al. Immune mimicry in malaria: Plasmodium falciparum secretes a functional histamine-releasing factor homolog in vitro and in vivo. Proc Natl Acad Sci USA $2001 ; 98: 10829-32$.

24. Beghdadi W, Porcherie A, Schneider BS, et al. Inhibition of histamine-mediated signaling confers significant protection against severe malaria in mouse models of disease. J Exp Med $2008 ; 205: 395-408$.

25. Abbott NJ. Inflammatory mediators and modulation of blood-brain barrier permeability. Cell Mol Neurobiol $2000 ; 20: 131-47$.

26. Medana IM, Turner GD. Human cerebral malaria and the blood-brain barrier. Int J Parasitol $2006 ; 36: 555-68$.

27. Belnoue $\varepsilon$, Kayibanda M, Vigario AM, et al. On the pathogenic role of brain-sequestered alphabeta CD8 ${ }^{+}$T cells in experimental cerebral malaria. J Immunol $2002 ; 169$ : 6369-75.

28. Yanez DM, Manning DD, Cooley AJ, et al. Participation of lymphocyte subpopulations in the pathogenesis of experimental murine cerebral malaria. J Immunol 1996; 157 : 1620-4.

29. Bryce PJ, Mathias $\mathrm{CB}$, Harrison $\mathrm{KL}$, et al. The $\mathrm{Hl}$ histamine receptor regulates allergic lung responses. J Clin Invest $2006 ; 116: 1624-32$.

30. Favre N, Da Laperousaz C, Ryffel B, et al. Role of ICAM-1 (CD54) in the development of murine cerebral malaria. Microbes Infect 1999; 1:961-8.

31. Kimura S, Wang Ky, Tanimoto A, et al. Acute inflammatory reactions caused by histamine via monocytes/macrophages chronically participate in the initiation and progression of atherosclerosis. Pathol Int $2004 ; 54: 465-74$.

32. Shimizu T, Nishihira J, Watanabe $H$, et al. Cetirizine, an Hl-receptor antagonist, suppresses the expression of macrophage migration inhibitory factor : its potential anti-inflammatory action. Clin Exp Allergy $2004 ; 34: 103-9$.

33. Baunaure F, Langsley G. Trafic protéique dans le globule rouge infecté par Plasmodium. Med Sci (Paris) $2005 ; 21: 523-9$.
TIRÉS À PART

S. Mécheri 


\section{Ateliers de formation 2009}

Renseignements et inscriptions :

Ateliers de formation Inserm

101, rue de Tolbiac

75654 Paris Cedex 13

Tél. : 33 (0)1 44236204 - Fax : 33 (0)1 44236293

\section{Atelier de formation $\mathrm{n}^{\circ} 198$}

\section{Protocoles récents en épidémiologie}

Organisateurs : Nadine Andrieu (Inserm U900, Paris), Michel Chavance (Inserm U780, Villejuif), Pascal Wild (Nancy)

\section{Phase I • Le point sur...}

30 septembre-2 octobre 2009

Objectifs - Présenter de nouveaux schémas d'enquêtes épidémiologiques développés ces dernières années : études limitées aux cas et plans d'échantillonnage complexe optimisés, expliquer les modèles d'analyse à mettre en œuvre.

Public • Épidémiologistes particulièrement intéressés par les problèmes méthodologiques et biostatisticiens débutants ou confirmés.

Chercheurs, ingénieurs ou techniciens.

Les présentations seront effectuées en anglais.

Nombre maximum de participants : 60

Programme - Études limitées aux cas : cas croisés, séries de cas. Double échantillonnage simple ou stratifié : cas-témoins emboîtés dans une cohorte, cas-cohorte, études en deux phases et contre-appariement. Les exemples présentés concerneront en particulier la pharmacoépidémiologie, l'épidémiologie du cancer, l'épidémiologie cardio-vasculaire, l'étude des interactions gènesenvironnement.

\section{Phase II - Maîtrise technique 5-7 octobre 2009 • Villejuif}

Programme - Présentation et discussion de programmes (SAS, STATA, R,...) adaptés à l'analyse d'enquêtes réalisées selon des protocoles présentés en phase I. Analyse de données fournies par les participants.

Sélection • 12 personnes seront retenues parmi les participants de la phase I. Deux critères importants seront l'intérêt de leurs données et leur expérience statistique.

Avec la participation de - Nadine Andrieu (Paris, France), Jonine Bernstein (Yale, USA), Norman Breslow (Seattle, USA), Michel Chavance (Villejuif, France), Paddy Farrington (Milton Keynes, UK), Mounia Hocine (Villejuif, France), Bryan Langholz (Los Angeles, USA), Thomas Lumley (Seattle, USA), Helena Marti (Villejuif, France), Walter Schill (Bremmen, Deutschland), Pascal Wild (Nancy, France)

> Grâce à $\mathrm{m} / \mathrm{s}$, vous vivez en direct les progrès des sciences biologiques et médicales

Abonnez-vous

à Médecine/Sciences
Bulletin d'abonnement page 418 dans ce numéro de $\mathrm{m} / \mathrm{s}$

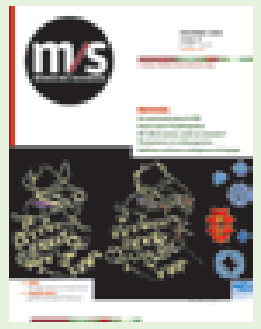

